\title{
Archéopages
}

Archéopages

Archéologie et société

Hors-série 3 | 2012

Nouveaux champs de la recherche archéologique

\section{Une enceinte néolithique à Carvin}

\section{Cécile Monchablon}

\section{(2) OpenEdition}

\section{Journals}

Édition électronique

URL : https://journals.openedition.org/archeopages/688

DOI : 10.4000/archeopages.688

ISSN : 2269-9872

\section{Éditeur}

INRAP - Institut national de recherches archéologiques préventives

\section{Édition imprimée}

Date de publication : 1 janvier 2012

Pagination : 105-109

ISSN : 1622-8545

\section{Référence électronique}

Cécile Monchablon, « Une enceinte néolithique à Carvin », Archéopages [En ligne], Hors-série 3 | 2012,

mis en ligne le 01 janvier 2012, consulté le 27 février 2023. URL : http://journals.openedition.org/ archeopages/688 ; DOI : https://doi.org/10.4000/archeopages.688 
est discutée (Cottiaux, Robert, 1987 ; Dubouloz et al, 1996), de même que son appartenance à la culture du Cerny (Joseph et al., 2011).

Les bâtiments de Berry-au-Bac «La Croix Maigret» (Dubouloz et al., 1982) d'Osly-Courtil «La Terre-Saint-Mard» (Dubouloz, 200o) ont des plans rectangulaires ou légèrement trapézoïdaux d'une vingtaine de mètres de long pour une dizaine de large, à trois rangées de poteaux porteurs avec des cloisons latérales, plantées séparément ou installées dans une tranchée commune. Pour trouver des éléments comparatifs du Michelsberg, il faut se tourner vers le site de Mairy (Marolles, 1989), dans les Ardennes, ou vers le groupe voisin de Spiere, à Carvin. Leurs bâtiments reprennent l'organisation des plans post-Rössen, mais peuvent atteindre des tailles bien supérieures et possèdent des murs latéraux en tranchées profondes.

Les bâtiments trouvés récemment à Carvin «La Gare d'eau » (Monchablon et al., 2011) reprennent les caractéristiques générales de ceux de Mairy.

Excepté l'orientation identique, ces plans tranchent totalement avec ceux de Cuiry-lès-Chaudardes «Le Champ Tortu». Les plans rectangulaires sont remplacés par des plans polygonaux; les dimensions chutent à une dizaine de mètres et les poteaux centraux et les cloisons latérales disparaissent totalement. Ces distinctions sont peut-être d'ordre chronologique. Les premiers édifices appartiennent au post-Rössen ou au Michelsberg ancien, tandis que ceux de Cuiry datent du Michelsberg récent. Compte tenu de la date «tardive» des bâtiments $\mathrm{du}$ «Champ Tortu », il est tentant de les considérer comme des prototypes des plans en abside du Néolithique final, mais les données radiocarbones révèlent l'ancienneté des plans rectangulaires sur ceux en abside.

Le site Michelsberg de Cuiry-lès-Chaudardes «Le Champ Tortu» rassemble quatre à sept bâtiments. L'organisation en plan, les caractéristiques architectures communes et l'agencement similaire militent en faveur d'une certaine contemporanéité qui autorise à parler de village. Les plans des maisons, de forme polygonale avec une extrémité en abside, ne trouvent de comparaison ni dans ce qui précède ni dans ce qui succède. La disparition des poteaux faîtiers, des cloisons internes et des dimensions réduites contrastent totalement avec les quelques plans de bâtiments connus pour le début du Néolithique moyen II. Seule l'orientation est-ouest subsiste. Ces caractéristiques nouvelles sont peutêtre chronologiques. Létape du Michelsberg récent est, en effet, presque inconnue dans le Nord de la France. Mais aucun plan similaire ne semble exister, ni en Alsace, ni en Allemagne, où cette culture est mieux connue. Le caractère unique de ces bâtiments provient-il de l'état actuel de nos connaissances ou bien dénote-il d'une fonction particulière du site, difficile à appréhender pour le moment ? Ces réflexions seront à développer plus avant dans le cadre de la publication du site, mais il semble bien que, exceptée l'orientation, il ne reste plus rien du modèle architectural danubien qui a pourtant perduré pendant plusieurs siècles dans notre région et que l'on perçoit pourtant encore dans le bâtiment monumental de Beaurieux situé à quelques centaines de mètres (Colas et al., 2008) [cf. encadré p 101].

COLAS C., GRANSAR F., NAZE Y., à paraître : Le village du Michelsberg récent Cuiry-Lès-Chaudardes «Le Champ Tortu », Rapport final d'opération de fouilles, Inrap.

Colas C., Thevenet C. Manolakakis L., Maigrot Y., Auxiette G., Chartier M., Baillieu M., Naze Y., 2008 : Beaurieux (Aisne) La Plaine, zone sud, tranche 2, Rapport de fouille intermédiaire, Inrap, SRA Picardie, 105 p., 67 fig.

Cottiaux R., Robert B., 1987 : « Le site Néolithique et des Ages des Métaux de Berry-au-Bac "Le Vieux Tordoir" », Fouilles Protohistoriques de la Vallée de L'Aisne, 15, p. 86-106.

Dubouloz J., Ilett M., LASSERre M., 1982 : « Enceintes et maisons chalcolithiques de Berry-au-Bac, la Croix Maigret (Aisne) », Le Néolithique de l'Est de la France, Actes du colloque de Sens, Société Archéologique de Sens, 1, p. 193-206.

Dubouloz J., Farruggia J.-P., Robert B., 1996 : « Bâtiments néolithiques non-rubanés à Berry-au-Bac "Le Vieux Tordoir", Aisne : présentation préliminaire », Internéo, p. 51-69.

Joseph F., Julien M., Leroy-Langelin E., Lorin Y. Praud Y., 2011 : « L'architecture domestique des sites du $\mathrm{III}^{\mathrm{e}}$ millénaire av. notre ère dans le Nord de la France. Le Néolithique du Nord de la France dans son contexte européen. Habitat et économie aux $4{ }^{\mathrm{e}}$ et $3^{\mathrm{e}}$ millénaires av. notre ère », Revue Archéologique de Picardie, $\mathrm{n}^{\circ}$ spécial 28, p. 249-272.

MAROLle C., 1989 : « Le village Michelsberg des hautes Chanvières à Mairy », Gallia préhistoire, t. 31, p. 93-118.

Monchablon C., Baillieu M., Bouchet M., Goutelard A., Praud I., 2011, «L'enceinte Néolithique moyen II de Carvin "La Gare d'Eau" (Pas-de-Calais). Présentation préliminaire », in Bostyn F., Martial E., Praud I. (DIR), Le Néolithique du nord de la France dans son contexte européen. Habitat et économie au 4e et $3 e$ millénaires av. notre ère, Revue Archéologique de Picardie, n spécial, 28, p. 407-417.

ZeEb-LANZ A., 2001: «Maisons semi-excavées en Allemagne du SudOuest : un mode d'habitat caractéristique pour le début du Néolithique récent? ", Bulletin de la Société Préhistorique Française, t. $98, n^{\circ} 2$, p. $285-297$.

\section{Une enceinte néolithique à Carvin}

\section{Cécile Monchablon}

Inrap, UMR 7041 «Protohistoire européenne »

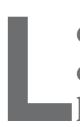
e site est implanté à proximité immédiate de la Deûle et sur une butte bordée de zones humides. L'enceinte entoure cette butte en un système organisé de fossés. Deux palissades (dont l'une en tranchée) et deux fossés discontinus se déploient de façon concentrique sur une surface de 5 hectares environ. Un troisième fossé extérieur, dont le tracé ne suit pas l'arrondi général, s'ajoute à ce système. Dans le paysage, aux excavations larges et profondes des fossés faisaient écho les levées de terre issues de leur creusement. Les deux palissades, hautes de plusieurs mètres, ont nécessité, pour leur construction, l'abattage et l'équarrissage de plusieurs centaines d'arbres. Fossés et palissades s'interrompent fréquemment au même rythme, constituant ainsi de véritables points de passage entre extérieur et intérieur de l'enceinte.

Dans la surface enclose, outre des fosses néolithiques (fosses-dépotoirs, structures de stockage...), deux bâtiments partiellement conservés ont été identifiés. Ils mesurent une vingtaine de mètres de long et 7 à $8 \mathrm{~m}$ de large. Un axe central de poteaux était destiné à porter le faîtage d'un toit à deux pans. L'espace interne était compartimenté par des fosses oblongues perpendiculaires, dans lesquelles étaient logés deux à trois poteaux. Ces parois n'ont pas toujours été reconnues : elles correspondent à des fondations sur sablières difficilement repérables aujourd'hui. 

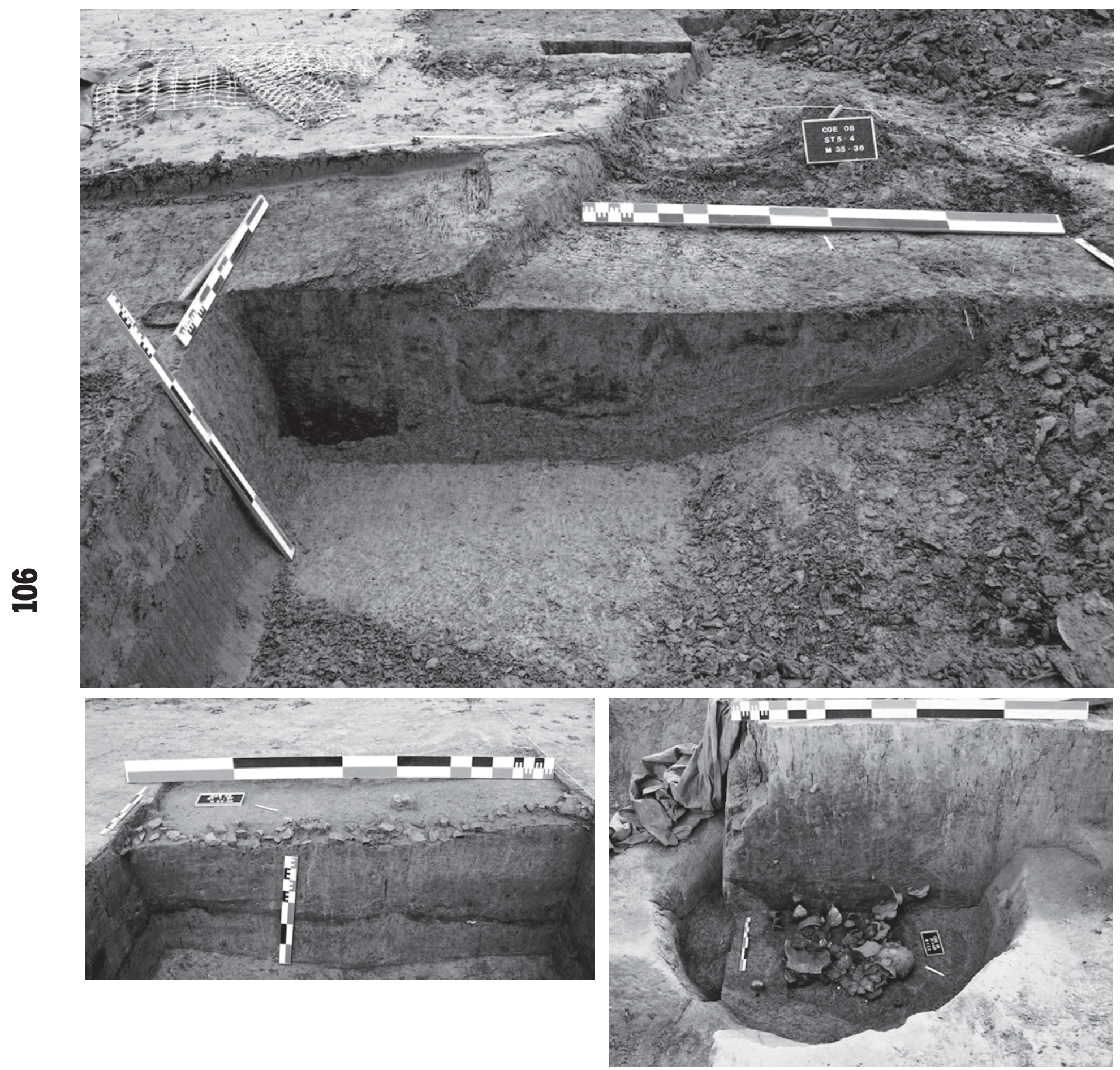

[Fig.1] Palissade en tranchée avec

poteaux équarris implantés à

différentes profondeurs.

[Fig.2] Rejets céramiques au sommet d'un fossé.

[Fig.3] Dépôt au fond d’un fossé.

[Fig.4] Diverses phases de comblement et de re-creusement danstundes fossés.

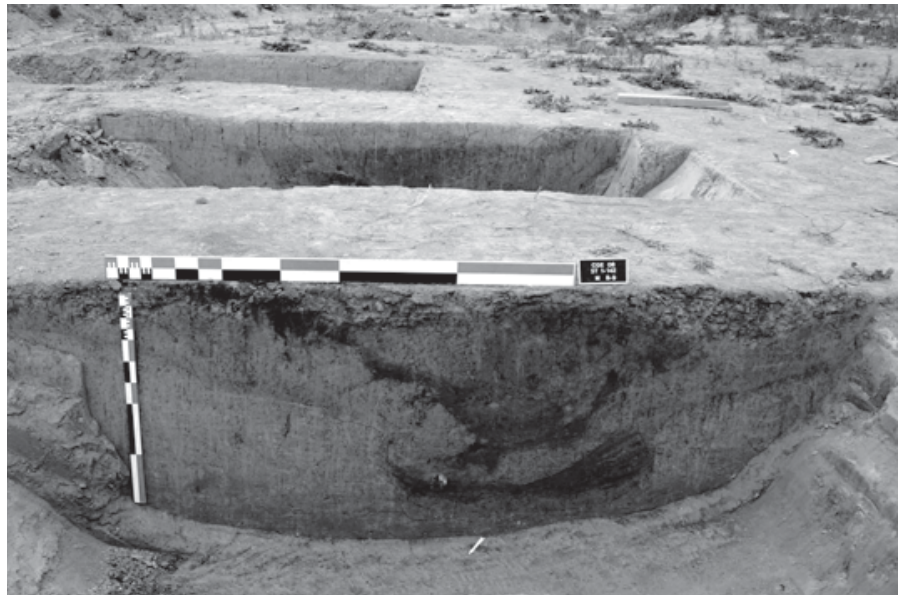


Ces édifications (poteaux équarris de forme rectangulaire pour l'une des palissades [Fig.1] et maisons à une rangée axiale de poteaux porteurs) suggèrent une gestion rigoureuse et maîtrisée du travail du bois, ainsi que l'invention de techniques nouvelles pour l'assemblage des charpentes : tenon- mortaise et chevillage.

L'ensemble des structures a livré une grande quantité de mobilier (surtout lithique et céramique) qui place le site de Carvin dans le groupe chronoculturel de Spiere, groupe contemporain du Chasséen septentrional et du Michelsberg. Certains objets confirment l'existence de réseaux d'échanges : quelques outils lithiques sont en silex originaire des minières de Spiennes (Hainaut belge), ou encore un pendentif en fluorite provient de gisements dont les plus proches connus se situent aux alentours de Charleroi (Hainaut belge également). Dans les fossés, des zones de rejet [fig. 2] se distinguent des zones de dépôt où le mobilier a été « mis en scène » avec des céramiques souvent entières [fig. 3-4].

Outre la question de la durée d'utilisation pour ce type de site, se pose celle de sa fonction : simple habitat fortifié ou place centrale d'un territoire organisé en groupe de fermes et de villages?

$[\ldots]$

Les occupations de la fin du Néolithique.

Les dernières phases correspondant à la fin du Néolithique (3600-2 ooo av. notre ère) restaient jusque très récemment, parmi les plus mal connues dans toute la moitié nord de la France. En effet, c'est principalement par l'étude des sépultures collectives qu'elles étaient appréhendées (donc par des fouilles souvent anciennes et un mobilier spécifique à ce type de structures). Nos connaissances sont également fondées sur des enceintes dans le centre et centreouest de la France, et ailleurs sur des occupations comprenant des fosses domestiques isolées, un peu de matériel piégé dans les couches de sédiment, ainsi qu'un corpus limité de mobilier ne permettant pas de se faire une idée exacte du mode vie des populations et des modalités de leur implantation.

Aussi, les différentes occupations de la fin du IV ${ }^{\mathrm{e}}$ millénaire av. notre ère de Pont-sur-Seine (Yonne) [cf. encadré p 109], fouillées sur quelques 8 hectares, avec ses trois enclos comprenant une douzaine de bâtiments qui sont peut-être des habitations et deux architectures monumentales exceptionnelles dont les fondations sont en pierre, constituent-t-elles une avancée importante dans la connaissance de cette période.

Peu après, à la fin du Néolithique apparaissent, sur tout le territoire, de grands bâtiments collectifs dépassant parfois les 100 m de longueur, tels Moulinssur Céphons (Indre-et-Loire) ou Pléchatel (Illeet-Vilaine). À Houplin-Ancoisne (Pas-de-Calais), un bâtiment dépasse les $40 \mathrm{~m}$ de longueur [cf. encadré p 111]. Ces dernières années, plusieurs architectures de ce type, mais de dimensions moins importantes, ont également été révélées pour la première fois dans le Nord de la France, dans des contextes variés, qu'il s'agisse de fonds de vallée et de plateaux limoneux, même si on constate une préférence pour les zones humides. Les bâtiments sont de plan rectangulaire, parfois terminés en abside ; leur orientation est souvent est-ouest, mais pas systématiquement, et ils peuvent être en aire ouverte ou entourés d'une enceinte. Dans le Sud-Ouest de la France, à Douchapt « Beauclair » (Dordogne), Pierrick Fouéré a mis au jour des longs bâtiments de $66 \mathrm{~m}$ et $50 \mathrm{~m}$ de longueur pour une largeur de $18 \mathrm{~m}$, de même orientation (est-ouest), divisés en 2 nefs par un alignement central de très gros poteaux placés à intervalles réguliers, tous les $9 \mathrm{~m}$, Ils sont partiellement ceinturés par un enclos en terre et semblent avoir été réalisés d'un seul tenant. Malgré ces variantes architecturales, ils s'inscrivent également dans une tradition d'architecture monumentale à l'instar des exemples cités précédemment. Outre ces architectures de grandes dimensions, quelques découvertes de sites d'habitats viennent corriger les irrégularités documentaires. Des structures plus légères, associant des aires d'activités spécialisées avec foyers à des trous de poteau et des fosses dépotoirs comme à Trémonteix (Puy-de-Dôme) [cf. encadré p 113] où des lambeaux de sol sont préservés. À Bédoin «Limon-Raspail » (Vaucluse) (Cauliez et al., 2011), un site de plein air en altitude (2 878-2 626 av. notre ère) comprend des vestiges de murs en terre crue et des torchis qui présentent des traces de négatifs de clayonnage, des silos, des structures de combustion et des fosses-dépotoirs. Tous ces sites contribuent à combler les hiatus importants pour la fin du Néolithique, tant dans le Sud que le Nord de la France.

En 2 oo9, nous déplorions le faible niveau de redondance des sites néolithiques mis au jour dans le cadre particulier et limité de l'Île-de-France (Samzun et al.,2009), et ce constat aurait très bien pu être établi dans d'autres régions françaises au cas par cas. À l'échelle nationale, ces lacunes se comblent progressivement comme nous venons de le voir. La documentation produite par l'archéologie préventive pour l'époque néolithique est à ce jour absolument considérable. L'exploitation de ces données commence à peine, même si la publication de certains sites majeurs est déjà réalisée ou enclenchée. De nombreux autres sites d'habitat sont cependant encore largement inédits. Nous faisons donc face à un double défi : d'une part, il est nécessaire d'assimiler un important corpus documentaire de façon à élaborer des synthèses interrégionales et à définir le cadre chronologique et culturel de ces découvertes qui a largement évolué ces dernières années. D'autre part, il est impératif de tenter d'appréhender la variabilité des habitats mis au jour, notamment leur hiérarchisation, afin d'aborder les questions ayant trait à l'organisation des sociétés néolithiques et à leur évolution. C'est bien entendu le souhait que nous formulons pour les dix prochaines années de l'Inrap.

BOSTYN F. (DIR.), 2003 : Néolithique ancien en Haute-Normandie : le village Villeneuve-Saint-Germain de Poses « Sur la Mare» et les sites de la boucle de Vaudreuil, Société préhistorique française, t. 4, travaux 4, 343 p. 


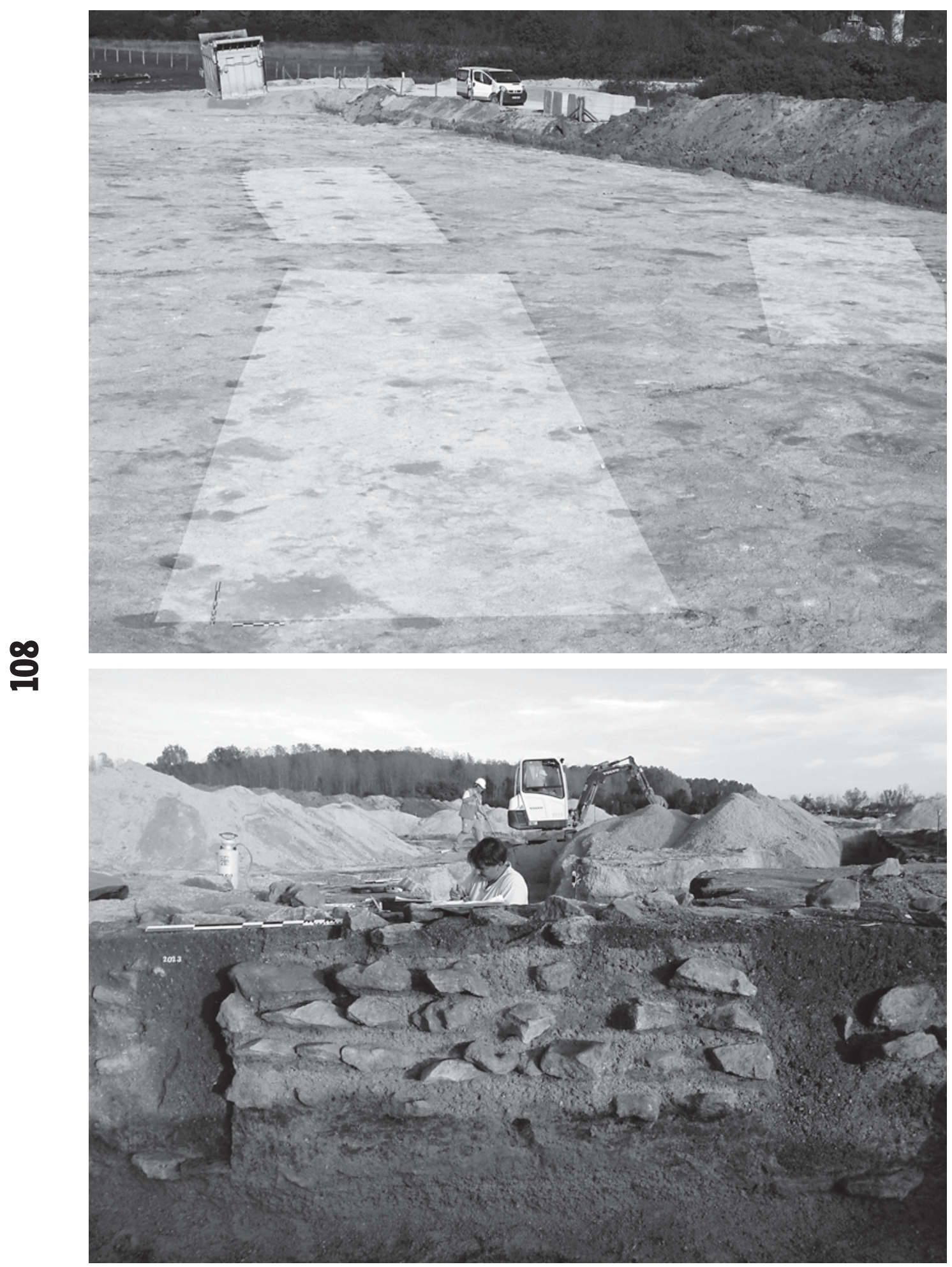

[Fig.1] Vue de bâtiments d'habitation [Fig.2] Vue des fondations de l'entrée datés de la $2^{\mathrm{e}}$ moitié du IV $\mathrm{e}^{\mathrm{e}}$ millénaire. de la construction monumentale. 
Cauliez, J., Blaise E., Bressy, Convertini F., Gilabert C., Hamon C., Lazard N., Negroni S., Ollivier V., Pelissier M., Petrequin, P., Piatschek C., Provenzano N., Renault S., 2011 : « Le site du Limon-Raspail à Bédoin dans le Vaucluse et le Néolithique final de moyenne vallée du Rhône », Bulletin de la Société préhistorique française, t. $108, \mathrm{n}^{\circ}$ 2, p. 263-330.

Creusillet M.-F., IrRibaria R., 2008: « Données récentes sur le Villeneuve Saint-Germain du sud-ouest du Bassin parisien », in BuRNEZ-LANOTTE L., Ilett M., Allard P., Fin des traditions danubiennes dans le Néolithique du Bassin parisien et de la Belgique (5100-470o av. J.-C.), Société préhistorique française, Mémoire XLIV, p. 161-18o.

Demoule J.-P., 2007 : « La révolution néolithique », Paris, La Découverte.

Dubouloz J., 2008 : « Beaucoup de sable et un peu de terre noire», Archéopages, hors-série, p. 49-55.

Dupont F., Liagre J., IrRibaria R., 2010 : « Sours les Ouches (Eure-etLoir). Un site original du Villeneuve-Saint-Germain ancien en région centre », in Billard C., Legris M. (DIR.), Premiers Néolithiques de l'Ouest. Cultures, réseaux, échanges des premières sociétés néolithiques à leur expansion, p. 75-10o.

Durand J., Bemilli C., Boitard-Bidault E., Cayol N., DietschSellami M.-F., Durand S., Fechner K., Maigrot Y., Praud I., 2008 : « Difficulté d'identification des sites du Néolithique ancien en contexte de plateau : l'exemple du site de Lieusaint "Centre Commercial 2" (Seine-et-Marne) », in Praud I., Giligny F. (DIR.), Internéo, 7, Société préhistorique française, p. 7-22

Ghesquiere E., MARCigny C., 2003 : « L'habitat du Néolithique moyen I de Vivoin » Le Parc » (Sarthe), Bulletin de la Société préhistorique française, t. $100, \mathrm{n}^{\circ}$ 3, p. 533-573.

LANCHON Y. (DIR.), 2009: «Le site de Changis « Les Pétreaux » (Seineet-Marne), Revue archéologique d'Ile-de-France, n 1, p. 43-92.

Marcigny C., Ghesquiere E., Juhel L., Charraud F, 2010 : « Entre Néolithique ancien et Néolithique moyen en Normandie et dans les îles anglo-normandes », in BILlard C., Legris M. (DIR.), Premiers Néolithiques de l'Ouest. Cultures, réseaux, échanges des premières sociétés néolithiques à leur expansion, p. 117-161.

Meunier K., SAmzUn A. : 2010 " Découverte d'un site du VSG ancien à Balloy "La Haute Borne" (Seine-et-Marne). Présentation des structures ", in Praud I., Besse M. (DIR.), Internéo, 8, Paris, p. 35-46.

Pailler Y., Marchand G., Blanchet S., Guyodo J., Hamon G., 2008: «Le Villeneuve-Saint-Germain dans la péninsule Armoricaine : les débuts d'une enquête », in Burnez-Lanotte L., Ilett M., Allard P., Fin des traditions danubiennes dans le Néolithique du Bassin parisien et de la Belgique (5100-4700 av. J.-C.), Société préhistorique française, Mémoire, XLIV, p. 91-111.

Praud I., Bostyn F., Cayol N., Hamon C., Ladureau, Lanchon Y., PinARD E., 2010 : « Entre Blicquy et Villeneuve-Saint-Germain. Présentation de la fouille d'un habitat du Néolithique ancien à Loison-sous-Lens (Pas-de-Calais) ». in BiLLARd C., LEgris M. (DIR.), Premiers Néolithiques de l'Ouest. Cultures, réseaux, échanges des premières sociétés néolithiques à leur expansion, Presses universitaires de Rennes (coll. Archéologie et culture), p. 305-323.

Samzun, A., Cottiaux, R. LANChON Y., 2009: « Néolithique en Ile-deFrance : la révolution reste à faire ", Archéopages, hors-série, p. 124-126.

SAMZUN, A., WARMÉ N., 2008 : « Fours, foyers et structures de combustion au Néolithique ancien et Néolithique moyen I : l'exemple du site de Buthiers-Boulancourt (Seine-et-Marne) », in Praud I., Giligny F. (DIR.), Internéo, 7, Société préhistorique française, p. 31-46.

\section{Une occupation du Néolithique récent à Pont-sur-Seine}

\author{
Vincent Desbrosse \\ Inrap \\ Virginie Peltier \\ Inrap
}

L' habitat de la fin du IV ${ }^{\mathrm{e}}$ millénaire est mal connu dans le bassin parisien. En effet il est généralement identifié à partir de mobilier piégé dans des horizons limoneux ou par des structures éparses ; ces traces limitées ne permettent donc pas de réfléchir sur les types d'occupation (Salanova, 2004, p. 78). La découverte d'un site d'habitat structuré de cette période, avec des plans de bâtiments préservés, revêt donc un intérêt tout particulier.

À Pont-sur-Seine (Aube), Le Haut de Launoy, l'organisation de l'occupation du Néolithique récent, est encore perceptible grâce à la préservation des fossés dans lesquels étaient enchâssés les poteaux de bois des palissades. Trois grands ensembles se dégagent. Un premier enclos de 1,4 ha renferme des bâtiments, certains étant à usage d'habitation [Fig.1]. L'agencement des constructions dans cette aire se précisera avec le résultat des datations en cours, mais on peut d'ores et déjà noter que la palissade dicte l'orientation de certains bâtiments d'habitation à proximité du fossé. Le deuxième enclos, d'une superficie de o,4 ha, renferme les deux constructions monumentales [Fig.2] ; leurs dimensions, les matériaux employés, le soin apporté à leur réalisation révèlent le caractère exceptionnel de ces deux édifices. Enfin, le dernier vaste ensemble regroupe la douzaine de bâtiments retrouvés hors des enceintes, principalement à l'est et au sud. La préservation de ces plans de maisons, pour des époques où ils font généralement défaut, est l'une des autres particularités du site de Pont-sur-Seine.

Jusqu'à présent, dans le bassin parisien, un seul plan de bâtiment de la deuxième moitié du IV $^{\mathrm{e}}$ millénaire était connu. Fouillé sur les marges occidentales de l'Argonne à l'occasion des opérations d'archéologie préventive de la LGV Est, le site de Dampierre-le-Château (Marne), Liévaux, a livré les traces d'une habitation; elle a été datée de cette période à partir des charbons de bois contenus dans ses poteaux (Dugois et al., 2002, p. 10). En fournissant une vingtaine de plans de bâtiments, la fouille de Pont-sur-Seine contribue à combler le déficit d'informations. Mais, en l'absence de mobilier caractéristique, il a fallu recourir à des analyses au carbone 14, pour dater ces constructions. Les fondations des habitations dépassent rarement la vingtaine de centimètres sous le décapage. Avec une érosion plus forte ou dans des conditions d'observation moins favorables, seuls les fossés des palissades et les fondations des constructions monumentales auraient été retrouvés. L'enceinte la plus vaste aurait donc été « vide », cas de figure courant dans les enceintes contemporaines du Centre-Ouest; les fouilles récentes menées dans ce secteur (Ard et al., 2009) semblent remettre ce point en question. La faible profondeur des creusements des bâtiments n'est pas nécessairement liée à un fort arasement des vestiges. En effet sur le site de Dampierre-le-Château, où l'impact de l'érosion a été jugé faible, voire inexistant, la profondeur moyenne des poteaux est de $9,3 \mathrm{~cm}$ pour une paroi, et de 12,9 $\mathrm{cm}$ pour l'autre, sous 30 à 40 $\mathrm{cm}$ de terre végétale. L'axe faîtier est quant à lui un peu plus profond avec une moyenne de $27,4 \mathrm{~cm}$ (Dugois et al., 2002, p. 9).

L'occupation du Haut de Launoy est aussi caractérisée par la quasi-absence de fosses contenant un mobilier détritique du Néolithique récent. En revanche, des artefacts ont été piégés dans des niveaux limoneux sur le pourtour du site. Le recours à une mécanisation réfléchie a permis d'accroitre le volume de sédiments traité et donc de palier en partie la faible densité de mobilier. Néanmoins, une série de datations absolues sur du matériel sélectionné, issu de creusements, s'avère indispensable pour démêler l'écheveau de la chronologie. Enfin, et pour terminer par l'aspect 